\title{
Exposing Latent Mutual Exclusion by Work Automata
}

\author{
Kasper Dokter ${ }^{(凶)}$ and Farhad Arbab \\ Centrum Wiskunde \& Informatica, Amsterdam, Netherlands \\ $\{$ K.P.C.Dokter, Farhad.Arbab\}@cwi.nl
}

\begin{abstract}
A concurrent application consists of a set of concurrently executing interacting processes. Although earlier we proposed work automata to specify both computation and interaction of such a set of executing processes, a detailed formal semantics for them was left implicit. In this paper, we provide a formal semantics for work automata, based on which we introduce equivalences such as weak simulation and weak language inclusion. Subsequently, we define operations on work automata that simplify them while preserving these equivalences. Where applicable, these operations simplify a work automaton by merging its different states into a state with a 'more inclusive' state-invariant. The resulting state-invariant defines a region in a multidimensional real vector space that potentially contains holes, which in turn expose mutual exclusion among processes. Such exposed dependencies provide additional insight in the behavior of an application, which can enhance scheduling. Our operations, therefore, potentially expose implicit dependencies among processes that otherwise may not be evident to exploit.
\end{abstract}

\section{Introduction}

Shared resources in a concurrent application must be protected against concurrent access. Mutual exclusion protocols offer such protection by granting access to a resource only if no other process has access. Moreover, concurrent applications often require some of their tasks to execute in some specific order. It is customary to implement both mutual exclusion and execution order among (sub-)tasks by means of locks. This practice suffers from two main drawbacks: First, contention on the shared resources results in blocked processes, which may lead to idle processors. Second, lock implementations introduce overhead that can become significant when executed repeatedly.

Alternatively, smart scheduling of processes can also offer protection against concurrent access, without suffering from drawbacks of locks. Suppose we have a crystal ball that accurately reveals when each process accesses its resources and their proper order of execution. We can then use this information to synthesize a scheduler that executes the processes in the correct order and prevents concurrent access to shared resources by speeding up or slowing down the execution of each process. Locks now become redundant, and their overhead can be avoided. 
In practice we have no such crystal ball for such accurate predictions. We can, however, take a step in the right direction by imagining the picture that we would see, if we had one. In our previous paper, we formalized such picture by introducing work automata [4]. A work automaton consists of states and transitions. Variables, called jobs, measure progress of all processes in a concurrent application. Each state admits a boolean constraint over jobs, called a stateinvariant, that defines the amount of work that can be done before a process blocks. Each transition consists of three parts: (1) a set of ports, called a synchronization constraint, that defines access to resources; (2) a boolean constraint over jobs, called a guard, that defines the amount of work that must be done before a transition can be fired; and (3) a set of jobs, called a reset, that identifies the jobs whose progress must be reset to zero.

The original definition of work automata in [4] left state-invariants, resets, and the formal semantics of work automata implicit, as this simpler model adequately served the purpose of that paper. In the current work (Sect. 2), however, we extend the generality of the work automata model by introducing stateinvariants and explicit reset of jobs. We define the formal semantics of work automata by means of labeled transition systems.

Compositionality is one of the most important features of work automata. Many small work automata compose into a single large automaton that models the behavior of the complete application. In view of state space explosion, a large number of states in a work automaton complicates its analysis. In Sect.3, we show by means of an example that some large work automata can be simplified to their respectively "equivalent" single state work automata. The state-invariant of the single state of such a resulting automaton defines a region in a multidimensional real vector space. Geometric features of this region reveal interesting behavioral properties of the corresponding concurrent application. For example, (explicit or implied) mutual exclusion in an application corresponds to a hole in its respective region, and non-blocking executions correspond to straight lines through this region. Since straight lines are easier to detect than non-blocking executions, the geometric perspective provides additional insight into the behavior of an application. We postulate that such information may be used to develop a smart scheduler that avoids the drawbacks of locks.

Motivated by our example, we define in Sect. 3 two procedures, called translation and contraction, that simplify a given work automaton by minimizing its number of states. We define weak simulation of work automata, and provide conditions (Theorems 1 and 2) under which translation and contraction preserve weak simulation. In Sect. 4, we discuss related work, and in Sect. 5 we conclude and point out future work.

\section{Work Automata}

Work automata, introduced in [4], originate from the need to represent progressing parallel tasks as a single automaton. In this section, we define work automata, their semantics, and operators such as composition and hiding. Our current definition of work automata differs from the original definition in [4] in two ways. 
First, our current definition of work automata includes explicit resets, while the original definition left this implicit. In Sect. 3.2, we use explicit resets to define a shifting operator that simplifies work automata. Second, our current definition of work automata includes state-invariants, while the original definition left them implicit. We use our explicit state-invariants to simplify the semantics of work automata, to simplify the composition of work automata, and, in Sect.3.1, to allow for more compact representations of an automaton.

\subsection{Syntax}

Consider an application $A$ that consists of $n \geq 1$ concurrently executing processes $X_{1}, \ldots, X_{n}$. We measure the progress of each process $X_{i}$ in $A$ by a positive real variable $x_{i} \in \mathbb{R}_{+}$, called a job, and represent the current progress of application $A$ by a map $p: J \rightarrow \mathbb{R}_{+}$, where $J=\left\{x_{1}, \ldots, x_{n}\right\}$ is the set of all jobs in $A$. We regulate the progress using boolean constraints $\phi \in B(J)$ over jobs:

$$
\phi::=\top|\perp| x \sim n\left|\phi_{0} \wedge \phi_{1}\right| \phi_{0} \vee \phi_{1},
$$

with $\sim \in\{\leq, \geq,=\}, x \in J$ a job and $n \in \mathbb{N}_{0} \cup\{\infty\}$. We define satisfaction $p \models \phi$ of a progress $p: J \rightarrow \mathbb{R}_{+}$and a constraint $\phi \in B(J)$ by the following rules: $p \models x \sim n$, if $p(x) \sim n ; p \models \phi_{0} \wedge \phi_{1}$, if $p \models \phi_{0}$ and $p \models \phi_{1} ; p \models \phi_{0} \vee \phi_{1}$, if $p \models \phi_{0}$ or $p \models \phi_{1}$. The interface of application $A$ consists of a set of ports through which $A$ interacts with its environment via synchronous operations, each one involving a subset $N \subseteq P$ of its ports.

We define the exact behavior of a set of processes as a labeled transition system called a work automaton. The progress value $p(x)$ of job $x$ may increase in a state $q$ of a work automaton, as long as the state-invariant $I(q) \in B(J)$ is satisfied. A state-invariant $I(q)$ defines the amount of work that each process can do in state $q$ before it blocks. A transition $\tau=\left(q, N, w, R, q^{\prime}\right)$ allows the work automaton to reset the progress of each job $x \in R \subseteq J$ to zero and change to state $q^{\prime}$, provided that the guard, defined as synchronization constraint $N \subseteq P$ together with the job constraint $w \in B(J)$, is satisfied. That is, the transition can be fired, if the environment is able to synchronize on the ports $N$ and the current progress $p: J \rightarrow \mathbb{R}_{+}$of $A$ satisfies job constraint $w$.

Definition 1 (Work automata). A work automaton is a tuple $(Q, P, J, I, \rightarrow$ $\left., \phi_{0}, q_{0}\right)$ that consists of a set of states $Q$, a set of ports $P$, a set of jobs $J$, a state invariant $I: Q \rightarrow B(J)$, a transition relation $\rightarrow \subseteq Q \times 2^{P} \times B(J) \times 2^{J} \times Q$, an initial progress $\phi_{0} \in B(J)$, and an initial state $q_{0} \in Q$.

Example 1 (Mutual exclusion). Figure 1 shows the work automata of two identical processes $A_{1}$ and $A_{2}$ that achieve mutual exclusion by means of a global lock $L$. The progress of process $A_{i}$ is recorded by its associated job $x_{i}$, and the interface of each process $A_{i}$ consists of two ports $a_{i}$ and $b_{i}$. Suppose we ignore the overhead of the mutual exclusion protocol. Then, lock $L$ does not need a job and its interface consists of ports $a_{1}, a_{2}, b_{1}$, and $b_{2}$. Each process $A_{i}$ starts in 


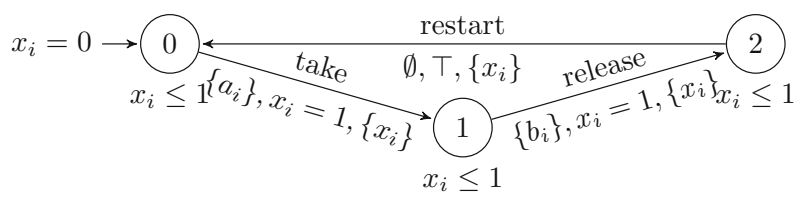

(a) Process $A_{i}$

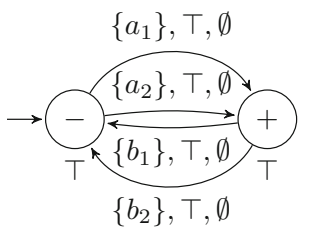

(b) Lock $L$

Fig. 1. Mutual exclusion of processes $A_{1}$ and $A_{2}$ by means of a lock $L$.

state 0 with $\phi_{0}:=x_{i}=0$ and is allowed to execute at most one unit of work, as witnessed by the state-invariant $x_{i} \leq 1$. After finishing one unit of work, $A_{i}$ starts to compete for the global lock $L$ by synchronizing on port $a_{i}$ of lock $L$. When $A_{i}$ succeeds in taking the lock, then lock $L$ changes its state from - to + and process $A_{i}$ moves to state 1 , its critical section, and resets the progress value of job $x_{i}$ to zero. Next, process $A_{i}$ executes one unit of work in its critical section. Finally, $A_{i}$ releases lock $L$ by synchronizing on port $b_{i}$, executes asynchronously its last unit of work in state 2 , and resets to state 0 .

\subsection{Semantics}

We define the semantics of a work automaton $A=\left(Q, P, J, I, \rightarrow, \phi_{0}, q_{0}\right)$ by means of a finer grained labeled transition system $\llbracket A \rrbracket$ whose states are configurations:

Definition 2 (Configurations). A configuration of a work automaton $A$ is a pair $(p, q) \in \mathbb{R}_{+}^{J} \times Q$, where $p: J \rightarrow \mathbb{R}_{+}$is a state of progress, and $q \in Q$ a state.

The transitions of $\llbracket A \rrbracket$ are labeled by two kinds of labels: one for advancing progress of $A$ and one for changing the current state of $A$. To model advance of progress of $A$, we use a map $d: J \rightarrow \mathbb{R}_{+}$representing that $d(x)$ units of work has been done on job $x$. Such a map induces a transition

$$
(p, q) \stackrel{d}{\rightarrow}(p+d, q)
$$

where + is component-wise addition of maps (i.e., $(p+d)(x)=p(x)+d(x)$, for all $x \in J)$. Figure 2(a) shows a graphical representation of transition (2). A state of progress $p$ of $A$ corresponds to a point in the plane.

In practice, the value of each job $x \in J$ continuously evolves from $p(x)$ to $p(x)+d(x)$. We assume that, during transition (2), each job makes progress at a constant speed. This allows us to view the actual execution as a path $\gamma:[0,1] \rightarrow \mathbb{R}_{+}^{J}$ defined by $\gamma(c)=p+c \cdot d$, where $\mathbb{R}_{+}^{J}$ is the set of maps from $J$ to $\mathbb{R}_{+}$and $\cdot$ is component-wise scalar multiplication (i.e., $(p+c \cdot d)(x)=p(x)+c \cdot d(x)$, for all $x \in J)$. At any instant $c \in[0,1]$, the state of progress $p+c \cdot d$ must satisfy the current state-invariant $I(q)$. Figure 2(a) shows execution $\gamma$ as the straight line connecting $p$ and $p+d$. For every $c \in[0,1]$, state of progress $\gamma(c)=p+c \cdot d$ corresponds to a point on the line from $p$ to $p+d$. Note that, since we have a 
transition from $p$ to $p+c \cdot d$ in $\llbracket A \rrbracket$ for all $c \in[0,1]$, Fig. 2(a) provides essentially a finite representation of an infinite semantics, i.e., one with an infinite number of transitions through intermediate configurations between $(p, q)$ and $(p+d, q)$. In Sect. 3.1, we use this perspective to motivate our gluing procedure.

The transition in (2) is possible only if the execution does not block between $p$ and $p+d$, i.e., state of progress $p+c \cdot d$ satisfies the state-invariant $I(q)$ of $q$, for all $c \in[0,1]$. Since $I(q)$ defines a region $\left\{p \in \mathbb{R}_{+}^{J} \mid p \models I(q)\right\}$ of a $|J|$-dimensional real vector space, the non-blocking condition just states that the straight line $\gamma$ between $p$ and $p+d$ is contained in the region defined by $I(q)$ (see Fig. 2(a)).

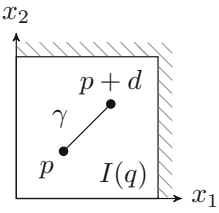

(a) Progress

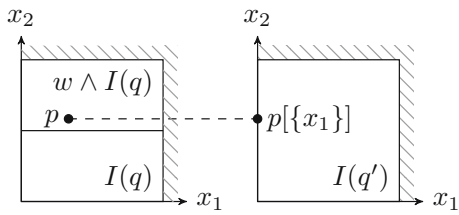

(b) State transition with reset

Fig. 2. Progress (a) of the application along the path $\gamma$ in $I(q)$ from $p$ to $p+d$, and (b) transition from state $q$ to $q^{\prime}$ with reset of job $x_{1}$.

A transition $\tau=\left(q, N, w, R, q^{\prime}\right)$ changes the state of the current configuration from $q$ to $q^{\prime}$, if the environment allows interaction via $N$ and the current state of progress $p$ satisfies job constraint $w$. As a side effect, the progress of each job $x \in R$ resets to zero. Such state changes occur on transitions of the form

$$
(p, q) \stackrel{N}{\longrightarrow}\left(p[R], q^{\prime}\right),
$$

where $p[R](x)=0$, if $x \in R$, and $p[R](x)=p(x)$ otherwise. Figure 2(b) shows a graphical representation of transition (3). The current state of progress satisfies both the current state-invariant and the guard of the transition, which allows to change to state $q^{\prime}$ and reset the value of $x_{1}$ to zero. For convenience, we allow at every configuration $(p, q)$ an $\emptyset$-labeled self loop which models idling.

Definition 3 (Operational semantics). The semantics of a given work automaton $A=\left(Q, P, J, I, \rightarrow, \phi_{0}, q_{0}\right)$ is the labeled transition system $\llbracket A \rrbracket$ with states $(p, q) \in \mathbb{R}_{+}^{J} \times Q$, labels $\mathbb{R}_{+}^{J} \cup 2^{P}$, and transitions defined by the rules:

$$
\begin{gathered}
\frac{d: J \rightarrow \mathbb{R}_{+}, \quad \forall c \in[0,1]: p+c \cdot d \models I(q)}{(p, q) \stackrel{d}{\rightarrow}(p+d, q)} \\
\frac{\tau=\left(q, N, w, R, q^{\prime}\right) \in \rightarrow, \quad p \models w \wedge I(q), \quad p[R] \models I\left(q^{\prime}\right)}{(p, q) \stackrel{N}{\rightarrow}\left(p[R], q^{\prime}\right)} \\
\frac{\emptyset}{(p, q) \stackrel{\emptyset}{\rightarrow}(p, q)}
\end{gathered}
$$

where $p[R](x)=0$, if $x \in R$, and $p[R](x)=p(x)$ otherwise. 


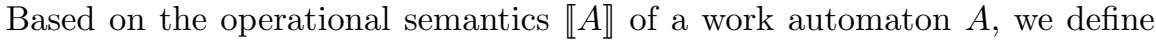
the trace semantics of a work automaton. The trace semantics defines all finite sequences of observable behavior that are accepted by the work automaton.

Definition 4 (Actions, words). Let $P$ be a set of ports and $J$ a set of jobs. An action is a pair $[N, d]$ that consist of a set of ports $N \subseteq P$ and a progress $d: J \rightarrow \mathbb{R}_{+}$. We write $\Sigma_{P, J}$ for the set of all actions over ports $P$ and jobs $J$. $W e$ call the action $[\emptyset, \mathbf{0}]$, with $\mathbf{0}(x)=0$ for all $x \in J$, the silent action. $A$ word over $P$ and $J$ is a finite sequence $u \in \Sigma_{P, J}^{*}$ of actions over $P$ and $J$.

Definition 5 (Trace semantics). Let $A=\left(Q, P, J, I, \rightarrow, \phi_{0}, q_{0}\right)$ be a work automaton. A run $r$ of $A$ over a word $\left(\left[N_{i}, d_{i}\right]\right)_{i=1}^{n} \in \Sigma_{P, J}^{*}$ is a path

$$
r:\left(p_{0}, q_{0}\right) \stackrel{N_{1}}{\longrightarrow} \stackrel{d_{1}}{\longrightarrow} s_{1} \quad \cdots \quad s_{n-1} \stackrel{N_{n}}{\longrightarrow} \stackrel{d_{n}}{\longrightarrow} s_{n}
$$

in $\llbracket A \rrbracket$, with $p_{0} \models \phi_{0} \wedge I\left(q_{0}\right)$. The language $L(A) \subseteq \Sigma_{P, J}^{*}$ of $A$ is the set of all words $u$ for which there exists a run of $A$ over $u$.

Example 2. The language of the process $A_{i}$ in Fig. 1(a) trivially contains the empty word, and the word $u=[\emptyset, \mathbf{1}][\{a\}, \mathbf{1}][\{b\}, \mathbf{1}]$, where $\mathbf{1}\left(x_{i}\right)=1$. Using Definitions 3 and 5 , we conclude that $v=[\emptyset, \mathbf{1}][\{a\}, \mathbf{1}][\{b\}, \mathbf{0 . 5}][\emptyset, \mathbf{0 . 5}]$, with $\mathbf{0 . 5}\left(x_{i}\right)=0.5$, is also accepted by $A_{i}$. Note that we can obtain $v$ from $u$ by splitting $[\{b\}, \mathbf{1}]$ into $[\{b\}, \mathbf{0 . 5}][\emptyset, \mathbf{0 . 5}]$.

\subsection{Weak Simulation}

Different work automata may have similar observable behavior. In this section, we define weak simulation as a formal tool to show their similarity. Intuitively, a weak simulation between two work automata $A$ and $B$ can be seen as a map that transforms any run of $A$ into a run of $B$ with identical observable behavior.

Following Milner [13], we define a new transition relation, $\Rightarrow$, on the operational semantics $\llbracket A \rrbracket$ of a work automaton $A$ that 'skips' silent steps.

Definition 6 (Weak transition relation). For any two configurations $s$ and $t$ in $\llbracket A \rrbracket$, and any $a \in \mathbb{R}_{+}^{J} \cup 2^{P}$ we define $s \stackrel{a}{\Rightarrow} t$ if and only if either

1. $a=\emptyset$ and $s(\stackrel{\emptyset}{\rightarrow})^{*} t$; or

2. $a \in 2^{P} \backslash\{\emptyset\}$ and $s \stackrel{\emptyset}{\Rightarrow} s^{\prime} \stackrel{a}{\rightarrow} s^{\prime \prime} \stackrel{\emptyset}{\Rightarrow} t$; or

3. $a \in \mathbb{R}_{+}^{J}, s \stackrel{\emptyset}{\Rightarrow} s_{1} \stackrel{c_{1} \cdot a}{\longrightarrow} t_{1} \stackrel{\emptyset}{\Rightarrow} s_{2} \cdots t_{n-1} \stackrel{\emptyset}{\Rightarrow} s_{n} \stackrel{c_{n} \cdot a}{\longrightarrow} t_{n} \stackrel{\emptyset}{\Rightarrow} t$, and $\sum_{i=1}^{n} c_{i}=1$,

with $n \geq 1, s_{i}, t_{i}$ configurations in $\llbracket A \rrbracket, c_{i} \in[0,1],\left(c_{i} \cdot a\right)(x)=c_{i} \cdot a(x)$, for all $x \in J$ and all $1 \leq i \leq n$.

Definition 7 (Weak simulation). Let $A_{i}=\left(Q_{i}, P, J, I_{i}, \rightarrow_{i}, \phi_{0 i}, q_{0 i}\right)$, for $i \in$ $\{0,1\}$ be two work automata, and let $\preceq \subseteq\left(\mathbb{R}_{+}^{J} \times Q_{0}\right) \times\left(\mathbb{R}_{+}^{J} \times Q_{1}\right)$ be a binary relation over configurations of $A_{0}$ and $A_{1}$. Then, $\preceq i s$ a weak simulation of $A_{0}$ in $A_{1}$ (denoted as $A_{0} \preceq A_{1}$ ) if and only if 
1. $p_{00} \models \phi_{00} \wedge I_{0}\left(q_{00}\right)$ implies $\left(p_{00}, q_{00}\right) \preceq\left(p_{01}, q_{01}\right)$, with $p_{01} \models \phi_{01} \wedge I_{1}\left(q_{01}\right)$; 2. $s \preceq t$ and $s \stackrel{a}{\rightarrow} s^{\prime}$, with $a \in \mathbb{R}_{+}^{J} \cup 2^{P}$, implies $t \stackrel{a}{\Rightarrow} t^{\prime}$ and $s^{\prime} \preceq t^{\prime}$, for some $t^{\prime}$.

We call $\preceq$ a weak bisimulation if and only if $\preceq$ and its inverse $\preceq^{-1}=\{(t, s) \mid$ $s \preceq t\}$ are weak simulations. We call $A_{0}$ and $A_{1}$ weakly bisimilar (denoted as $A_{0} \approx A_{1}$ ) if and only if there exists a weak bisimulation between them.

\subsection{Composition}

Thus far, our examples used work automata to define the exact behavior of a single job (or just a protocol $L$ in Fig. 1(b)). We now show that work automata are expressive enough to define the behavior of multiple jobs simultaneously. To this end, we define a product operator $\times$ on the class of all work automata. Before we turn to the definition, we first introduce some notation. For $i \in\{0,1\}$, let $A_{i}=$ $\left(Q_{i}, P_{i}, J_{i}, I_{i}, \rightarrow_{i}, \phi_{0 i}, q_{0 i}\right)$ be a work automaton and let $\tau_{i}=\left(q_{i}, N_{i}, w_{i}, R_{i}, q_{i}^{\prime}\right) \in$ $\rightarrow_{i}$ be a transition in $A_{i}$. We say that $\tau_{0}$ and $\tau_{1}$ are composable (denoted as $\left.\tau_{0} \frown \tau_{1}\right)$ if and only if $N_{0} \cap P_{1}=N_{1} \cap P_{0}$. If $\tau_{0} \frown \tau_{1}$, then we write $\tau_{0} \mid \tau_{1}=$ $\left(\left(q_{0}, q_{1}\right), N_{0} \cup N_{1}, w_{0} \wedge w_{1}, R_{0} \cup R_{1},\left(q_{0}^{\prime}, q_{1}^{\prime}\right)\right)$ for the composition of $\tau_{0}$ and $\tau_{1}$.

Definition 8 (Composition). Let $A_{i}=\left(Q_{i}, P_{i}, J_{i}, I_{i}, \rightarrow_{i}, \phi_{0 i}, q_{0 i}\right), i \in\{0,1\}$, be two work automata. We define the composition $A_{0} \times A_{1}$ of $A_{0}$ and $A_{1}$ as the work automaton $\left(Q_{0} \times Q_{1}, P_{0} \cup P_{1}, J_{0} \cup J_{1}, I_{0} \wedge I_{1}, \rightarrow, \phi_{00} \wedge \phi_{01},\left(q_{00}, q_{01}\right)\right)$, where $\rightarrow$ is the smallest relation that satisfies:

$$
\frac{i \in\{0,1\}, \tau_{i} \in \rightarrow_{i}, \tau_{1-i} \in \rightarrow_{1-i} \cup\left\{(q, \emptyset, \top, \emptyset, q) \mid q \in Q_{1-i}\right\}, \tau_{0} \frown \tau_{1}}{\tau_{0} \mid \tau_{1} \in \rightarrow}
$$

By means of the composition operator in Definition 8, we can construct large work automata by composing smaller ones. The following lemma shows that the composite work automaton does not depend on the order of construction.

Lemma 1. $\left(A_{0} \times A_{1}\right) \times A_{2} \approx A_{0} \times\left(A_{1} \times A_{2}\right), A_{0} \times A_{1} \approx A_{1} \times A_{0}$, and $A_{0} \times A_{0} \approx$ $A_{0}$, for any three work automata $A_{0}, A_{1}$, and $A_{2}$.

Example 3. Consider the work automata from Example 1. The behavior of the application is the composition $M$ of the two processes $A_{1}$ and $A_{2}$ and the lock $L$. Figure 3 shows the work automaton $M=L \times A_{1} \times A_{1}$. Each state-invariant equals $\top \wedge x_{1} \leq 1 \wedge x_{2} \leq 1$. The competition for the lock is visualized by the branching at the initial state 00 .

\section{$2.5 \quad$ Hiding}

Given a work automaton $A$ and a port $a$ in the interface of $A$, the hiding operator $A \backslash\{a\}$ removes port $a$ from the interface of $A$. As a consequence, the hiding operator removes every occurrence of $a$ from the synchronization constraint $N$ of every transition $\left(q, N, w, R, q^{\prime}\right) \in \rightarrow$ by transforming $N$ to $N \backslash\{a\}$. In case $N$ becomes empty, the resulting transition becomes silent. If, moreover, the source and the target states of a transition are identical, we call the transition idling. 


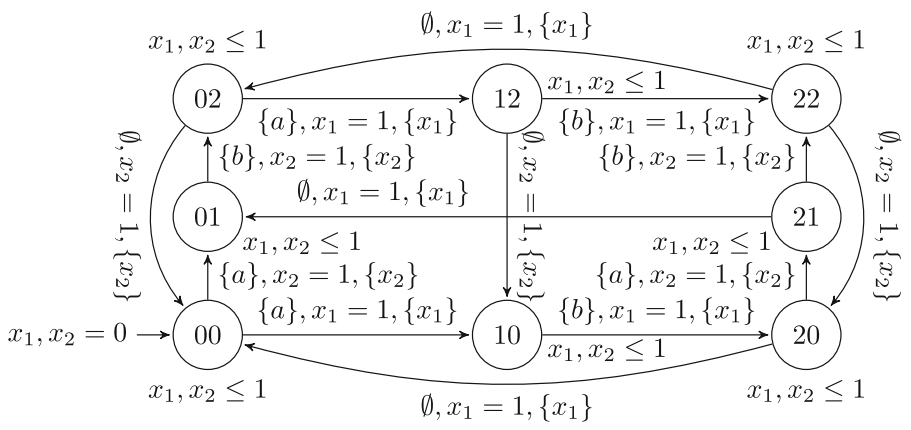

Fig. 3. The complete application $M=L \times A_{1} \times A_{2}$. In state $q_{1} q_{2}$, lock $L$ is in state $(-1)^{q_{1}+q_{2}+1}$ and process $A_{i}$ is in state $q_{i}$.

Definition 9 (Hiding). Let $A=\left(Q, P, J, I, \rightarrow, \phi_{0}, q_{0}\right)$ be a work automaton, and $M \subseteq P$ a set of ports. We define $A \backslash M$ as the work automaton $(Q, P \backslash$ $\left.M, J, \rightarrow_{M}, \phi_{0}, q_{0}\right)$, with $\rightarrow_{M}=\left\{\left(q, N \backslash M, w, R, q^{\prime}\right) \mid\left(q, N, w, R, q^{\prime}\right) \in \rightarrow\right\}$.

Lemma 2. Hiding partially distributes over composition: $M \cap P_{0} \cap P_{1}=\emptyset$ implies $\left(A_{0} \times A_{1}\right) \backslash M \approx\left(A_{0} \backslash M\right) \times\left(A_{1} \backslash M\right)$, for any two work automata $A_{0}$ and $A_{1}$ with interfaces $P_{0}$ and $P_{1}$, respectively.

Example 4. Consider the work automaton $M$ in Fig. 3. Work automaton $M^{\prime}=$ $M \backslash\{a, b\}$ is $M$ where every occurrence of $\{a\}$ or $\{b\}$ is substituted by $\emptyset$.

\section{State Space Minimization}

The composition operator from Definition 8 may produce a large complex work automaton with many different states. In this section, we investigate if, and how, a set of states in a work automaton can be merged into a single state, without breaking its semantics. In Sect.3.1, we present by means of an example the basic idea for our simplification procedures. We define in Sect. 3.2 a translation operator that removes unnecessary resets from transitions. We define in Sect. 3.3 a contraction operator that identifies different states in a work automaton. We show that translation and contraction are correct by providing weak simulations between their pre- and post-operation automata.

\subsection{Gluing}

The following example illustrates an intuitive gluing procedure that relates the product work automaton $M$ in Fig. 3 to the punctured square in Fig. 4(b). Formally, we define the gluing procedure as the composition of translation (Sect. 3.2) and contraction (Sect. 3.3). 


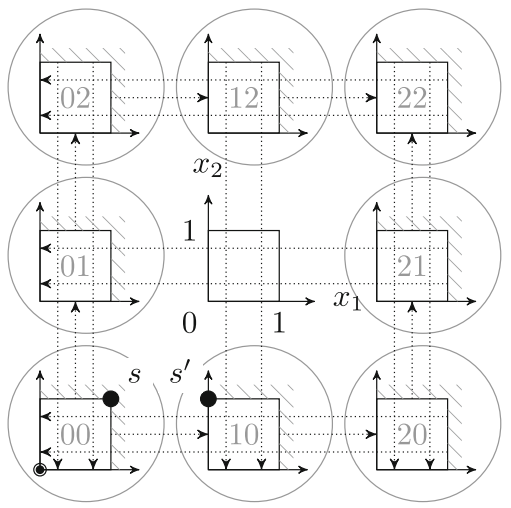

(a) $\llbracket M^{\prime} \rrbracket$

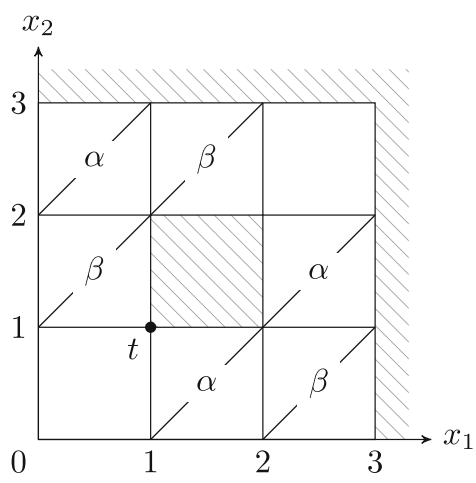

(b) $\llbracket K \rrbracket$

Fig. 4. Graphical representation (a) of semantics $\llbracket M^{\prime} \rrbracket$ of the work automaton $M^{\prime}$ in Example 4, where white regions represent state-invariants, and (b) result after gluing the regions in (a). Starting in a configuration below line $\alpha$ and above line $\beta$, parallel execution of $x_{1}$ and $x_{2}$ never blocks on lock $L$.

Example 5 (Gluing). Consider the work automaton $M^{\prime}$ in Example 4 that describes the mutual exclusion protocol for two processes. Our goal is to simplify $M^{\prime}$ to a work automaton $K$ that simulates $M^{\prime}$. To this end, we introduce in Fig. 4(a) a finite representation of the infinite semantics $\llbracket M^{\prime} \rrbracket$ of $M^{\prime}$, based on the geometric interpretation of progress discussed in Sect.2.2. For any given state $q$ of $M^{\prime}$, the state-invariant $I(q)=x_{1} \leq 1 \wedge x_{2} \leq 1$ is depicted in Fig. 4(a) as a region in the first quadrant of the plane. Each configuration $(p, q)$ of $M^{\prime}$ corresponds to a point in one of these regions: $q$ determines its corresponding region wherein point $p$ resides. Each transition of $M^{\prime}$ is shown in Fig. 4(a) as a dotted arrow from the border of one region to that of another region. We refer to these dotted arrows as jumps. A jump $\lambda$ from a region $R$ of state $q$ to another region $R^{\prime}$ of state $q^{\prime}$ represents infinitely many transitions from configurations $(p, q)$ to configurations $\left(p^{\prime}, q^{\prime}\right)$, for all $p$ and $p^{\prime}$, as permitted by the semantics $\llbracket M^{\prime} \rrbracket$. By the job constraint of the transition corresponding to $\lambda, p$ and $p^{\prime}$ must lie on the borders of $R$ and $R^{\prime}$, respectively, that are connected by $\lambda$.

From a topological perspective, a jump from one region to another can be viewed as 'gluing' the source and target configuration of that jump. We can glue any two regions in Fig. 4(a) together by putting regions (i.e., state-invariants) of the source and the target states side by side to form a single state with a larger region. Each jump in Fig. 4(a) from a source to a target state corresponds to an idling transition (c.f., rule (S3) in Definition 3) within a single state. When we apply this gluing procedure in a consistent way to every jump in Fig. 4(a), we obtain a single state work automaton $K$ that is defined by a single large region, as shown in Fig.4(b). Figure 5 shows the actual work automaton that corresponds to this region. Note that the restart transition allows the state of progress to jump in Fig. 4(a) from configuration $((x, 1), i 2)$ to $((x, 0), i 0)$ and 


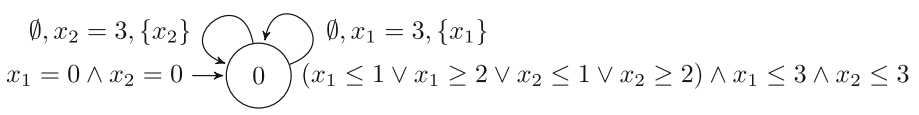

Fig. 5. Work automaton $K$ that corresponds to Fig. 4(b).

from configuration $((1, y), 2 j)$ to $((0, y), 0 j)$, for all $x, y \in[0,1]$ and $i, j \in\{0,1,2\}$. Thus, the restart transition identifies opposite boundaries in Fig. 4(b), turning the punctured square into a torus.

The next example shows that the geometric view of the semantics of the work automaton in Example 5 reveals some interesting behavioral properties of $M^{\prime}$.

Example 6. Consider the mutual exclusion protocol in Example 1. Is it possible to find a configuration such that parallel execution of jobs $x_{1}$ and $x_{2}$ (at identical speeds) never blocks, even temporarily, on lock $L$ ? It is not clear from the work automata in Fig. 1 (or in their product automaton as, e.g., in Fig. 3) whether such a non-blocking execution exists. Since only one process can acquire lock $L$, the execution that starts from the initial configuration blocks after one unit of work. However, using the geometric perspective offered by Fig. 4(b) and the fact that a parallel execution of jobs $x_{1}$ and $x_{2}$ at identical speeds correspond to a diagonal line in this representation, it is not hard to see that any execution path below line $\alpha$ and above line $\beta$ is non-blocking.

Regions of lock-free execution paths as revealed in Example 6 are interesting: if some mechanism (e.g., higher-level semantics of the application or tailor-made scheduling) can guarantee that execution paths of an application remains contained within such lock-free regions, then their respective locks can be safely removed from the application code. With or without such locks in an application code, a scheduler cognizant of such lock-free regions can improve resource utilization and performance by regulating the execution of the application such that its execution path remains in a lock-free region.

Example 7 (Correctness). Let $M^{\prime}$ be the work automaton in Example 4, and $K$ the work automaton in Fig. 5. We denote a configuration of $M^{\prime}$ as a tuple $\left(p_{1}, p_{2}, q_{0}, q_{1}, q_{2}\right)$, where $p_{i} \in \mathbb{R}_{+}$is the state of progress of job $x_{i}$, for $i \in\{0,1\}$, and $\left(q_{0}, q_{1}, q_{2}\right) \in\{-,+\} \times\{0,1,2\}^{2}$ is the state of $M^{\prime}$. We denote a configuration of $K$ as a tuple $\left(p_{1}, p_{2}, 0\right)$, where $p_{i} \in \mathbb{R}_{+}$is the state of progress of job $x_{i}$, for $i \in\{0,1\}$. The binary relation $\preceq$ over configurations of $M^{\prime}$ and $K$ defined by $\left(p_{1}, p_{2}, q_{0}, q_{1}, q_{2}\right) \preceq\left(q_{1}+p_{1}, q_{2}+p_{2}, 0\right)$, for all $0 \leq p_{i} \leq 1$ and $\left(q_{0}, q_{1}, q_{2}\right) \in$ $\{-,+\} \times\{0,1,2\}^{2}$, is a weak simulation of $M^{\prime}$ in $K$.

Note that $\preceq^{-1}$ is not a weak simulation of $K$ in $M^{\prime}$ due to branching. Consider the configurations $s=(1,1,-, 0,0)$ and $s^{\prime}=(0,1,+, 1,0)$ of $M^{\prime}$, and $t=(1,1,0)$ of $K$ (cf., Figs. 4(a) and (b)). While in configuration $t$ job $x_{2}$ can make progress, execution of $x_{2}$ is blocked at $s^{\prime}$ because process $A_{1}$ has obtained the lock. Since $s^{\prime} \preceq t$, we conclude that $\preceq^{-1}$ is not a weak simulation of $K$ in $M^{\prime}$. 
Fortunately, we can still prove that $K$ is a correct simplification of $M$ by transform $\preceq^{-1}$ into a weak simulation. Intuitively, such transformation remove pairs like $\left(t, s^{\prime}\right) \in \preceq^{-1}$. We make this argument formal in Sect. 3.3.

As illustrated in Example 6, gluing can reveal interesting and useful properties of an application. To formalize the gluing procedure, we define two operators on work automata. The main idea is to transform a given work automaton $A_{1}$ into an equivalent automaton $A_{2}$, such that (almost) any step $\left(p_{1}, q_{1}\right) \stackrel{\emptyset}{\rightarrow}\left(p_{1}^{\prime}, q_{1}^{\prime}\right)$ in $\llbracket A_{1} \rrbracket$ corresponds with an idling step $\left(p_{2}, q_{2}\right) \stackrel{\emptyset}{\rightarrow}\left(p_{2}^{\prime}, q_{2}^{\prime}\right)$ in $\llbracket A_{2} \rrbracket$, i.e., a step with $p_{2}^{\prime}=p_{2}$ and $q_{2}^{\prime}=q_{2}$. To achieve this correspondence, we define a translation operator that ensures $p_{2}^{\prime}=p_{2}$, and a contraction operator that ensures $q_{2}^{\prime}=q_{2}$.

\subsection{Translation}

In this section, we define the translation operator that allows us to remove resets of jobs from transitions. The following example shows that removal of job resets can be compensated by shifting the state-invariant of the target state.

Example 8 (Shifting). Suppose we remove the reset of job $x$ on the transition of work automaton $A$ in Fig. 6(a). If we fire the transition at $x=a \leq 1$, then the state of progress of $x$ in state 1 equals $a$ instead of 0 . We can correct this error by shifting the state-invariant of 1 by $a$, for every $a \leq 1$. We, therefore, transform the state-invariant of 1 into $x \leq 2$ (see Fig. 6(b)).

The transformation of work automata in Example 8 suggests a general translation procedure that, intuitively, (1) shifts each state-invariant $I(q), q \in Q$, along the solutions of some job constraint $\theta(q) \in B(J)$, and (2) removes for every transition $\tau=\left(q, N, w, R, q^{\prime}\right)$ some resets $\rho(\tau) \subseteq J$ from $R$.

Definition 10 (Shifts). A shift on a work automaton $\left(Q, P, J, I, \longrightarrow, \phi_{0}, q_{0}\right)$ is a tuple $(\theta, \rho)$ consisting of a map $\theta: Q \rightarrow B(J)$ and a map $\rho: \longrightarrow \longrightarrow 2^{J}$.

We define how to shift state-invariants along the solutions of a job constraint.

Definition 11. Let $\phi, \theta \in B(J)$ be two job constraints with free variables among $\mathbf{x}=\left(x_{1}, \ldots, x_{n}\right), n \geq 0$. We define the shift $\phi \uparrow \theta$ of $\phi$ along (the solutions of) $\theta$ as any job constraint equivalent to $\exists \mathbf{t}(\phi(\mathbf{x}-\mathbf{t}) \wedge \theta(\mathbf{t}))$.

Lemma 3. $\uparrow$ is well-defined: for all $\phi, \theta \in B(J)$ there exists $\psi \in B(J)$ such that $\exists \mathbf{t}(\phi(\mathbf{x}-\mathbf{t}) \wedge \theta(\mathbf{t})) \equiv \psi$.

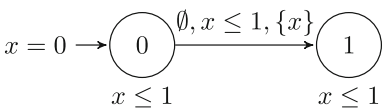

(a) $A$

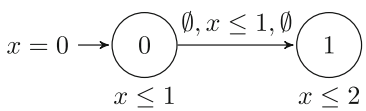

(b) $B$

Fig. 6. Shifting state-invariant $x \leq 1$ of state 1 in $A$ by one unit. 
We use a shift $(\theta, \rho)$ to translate guards and invariants along the solutions of job constraint $\theta$ and to remove resets occurring in $\rho$ :

Definition 12 (Translation). Let $\sigma=(\theta, \rho)$ be a shift on a work automaton $A=\left(Q, P, J, I, \rightarrow, \phi_{0}, q_{0}\right)$. We define the translation $A \uparrow \sigma$ of $A$ along the shift $\sigma$ as the work automaton $\left(Q, P, J, I_{\sigma}, \rightarrow_{\sigma}, \phi_{0} \uparrow \theta\left(q_{0}\right), q_{0}\right)$, with $I_{\sigma}(q)=I(q) \uparrow \theta(q)$ and $\rightarrow_{\sigma}=\left\{\left(q, N, w \uparrow \theta(q), R \backslash \rho(\tau), q^{\prime}\right) \mid \tau=\left(q, N, w, R, q^{\prime}\right) \in \rightarrow\right\}$.

Lemma 4. If $\theta \in B(J)$ has a unique solution $\delta \models \theta$, then $p+\delta \models \phi \uparrow \theta$ implies $p \models \phi$, for all $p \in \mathbb{R}_{+}^{J}$ and $\phi \in B(J)$.

Theorem 1. If $p=w \wedge I(q)$ and $\delta \models \theta(q)$ implies $(p+\delta)[R \backslash \rho(\tau)]-p[R] \models$ $\theta\left(q^{\prime}\right)$, for every transition $\tau=\left(q, N, w, R, q^{\prime}\right)$ and every $p, d \in \mathbb{R}_{+}^{J}$, then $A \preceq A \uparrow$ $\sigma$. If, moreover, $\theta(q)$ has for every $q \in Q$ a unique solution, then $A \approx A \uparrow \sigma$.

For at transition $\tau=\left(q, N, w, R, q^{\prime}\right)$, suppose $\theta(q)$ and $\theta\left(q^{\prime}\right)$ define unique solutions $\delta$ and $\delta^{\prime}$, respectively. If $\sigma$ eliminates job $x \in R$ (i.e., $x \in \rho(\tau)$ ), then $p(x)+\delta(x)=\delta^{\prime}(x)$, for all $p \models w \wedge I(q)$. Thus, $w \wedge I(q)$ must imply $x=$ $\delta^{\prime}(x)-\delta(x)$, which seems a strong assumption. For a deterministic application, however, it makes sense to have only equalities in transition guards. In this case, a transition is enabled only when a job finishes some fixed amount of work, which corresponds to having only equalities in transition guards.

Example 9. Let $M^{\prime}$ be the work automata in Example $4, \sigma=(\delta, \rho)$ the shift defined by $\theta(q):=x_{1}=q_{1} \wedge x_{2}=q_{2}$, and $\rho(\tau)=R_{\tau}$. Theorem 1 shows that $M^{\prime} \uparrow \sigma$ and $M^{\prime}$ are weakly bisimilar.

\subsection{Contraction}

In this section, we define a contraction operator that merges different states into a single state. To determine which states merge and which stay separate, we use an equivalence relation $\sim$ on the set of states $Q$.

Definition 13 (Kernel). A kernel of a work automaton $A$ is an equivalence relation $\sim \subseteq Q \times Q$ on the state space $Q$ of $A$.

Recall that an equivalence class of a state $q \in Q$ is defined as the set $[q]=$ $\left\{q^{\prime} \in Q \mid q \sim q^{\prime}\right\}$ of all $q^{\prime} \in Q$ related to $q$. The quotient set of $Q$ by $\sim$ is defined as the set $Q / \sim=\{[q] \mid q \in Q\}$ of all equivalence classes of $Q$ by $\sim$. By transitivity, distinct equivalence classes are disjoint and $Q / \sim$ partitions $Q$.

Definition 14 (Contraction). The contraction $A / \sim$ of a work automaton $A=\left(Q, P, J, I, \rightarrow, \phi_{0}, q_{0}\right)$ by a kernel $\sim$ is defined as $\left(Q / \sim, P, J, I^{\prime}, \rightarrow^{\prime}, \phi_{0},\left[q_{0}\right]\right)$, where $\rightarrow^{\prime}=\left\{\left([q], N, w, R,\left[q^{\prime}\right]\right) \mid\left(q, N, w, R, q^{\prime}\right) \in \rightarrow\right\}$ and $I^{\prime}([q])=\bigvee_{\tilde{q} \in[q]} I(\tilde{q})$.

The following results provides sufficient conditions for preservation of weak simulation by contraction. The relation $\preceq$ defined by $(p,[q]) \preceq(p, q)$, for all $(p, q) \in \mathbb{R}_{+}^{J} \times Q$, is not a weak simulation of $A / \sim$ in $A$. As indicated in Example 7 , we can restrict $\preceq$ and require only $(p,[q]) \preceq(p, \alpha(p,[q]))$, for some section $\alpha$. 
Definition 15 (Section). A section is a map $\alpha: \mathbb{R}_{+}^{J} \times Q / \sim \rightarrow Q$ such that for all $q, q^{\prime} \in Q$ and $p, d \in \mathbb{R}_{+}^{J}$

1. $p=I^{\prime}([q])$ implies $p \mid=I(\alpha(p,[q]))$;

2. $q \sim \alpha(p,[q])$

3. $p=\phi_{0} \wedge I\left(q_{0}\right)$ implies $\alpha\left(p,\left[q_{0}\right]\right)=q_{0}$;

4. $(p,[q]) \stackrel{N}{\longrightarrow}\left(p^{\prime},\left[q^{\prime}\right]\right)$ implies $(p, \alpha(p,[q])) \stackrel{N}{\Rightarrow}\left(p^{\prime}, \alpha\left(p^{\prime},\left[q^{\prime}\right]\right)\right)$;

5. $(p, q) \stackrel{d}{\rightarrow}(p+d, q)$ implies $(p, \alpha(p,[q])) \stackrel{d}{\Rightarrow}(p+d, \alpha(p+d,[q]))$.

In contrast with conditions (1), (2), and (3) in Definition 15, conditions (4) and (5) impose restrictions on the contraction $A / \sim$. These restrictions allow us to prove, with the help of the following lemma, weak simulation of $A / \sim$ in $A$.

Lemma 5. If $(p,[q]) \stackrel{d}{\rightarrow}(p+d,[q])$, then there exist $k \geq 1,0=c_{0}<\cdots<c_{k}=1$ and $q_{1}, \ldots, q_{k} \in[q]$ such that $p+c \cdot d \models I\left(q_{i}\right)$, for all $c \in\left[c_{i-1}, c_{i}\right]$ and $1 \leq i \leq k$.

Theorem 2. $A \preceq A / \sim$; and if there exists a section $\alpha$, then $A / \sim \preceq A$.

In our concluding example below, we revisit our intuitive gluing procedure motivated in Sect.3.1 to show how the theory developed in Sects. 3.2 and 3.3 formally supports our derivation of the geometric representation of $\llbracket K \rrbracket$ from $\llbracket M^{\prime} \rrbracket$ and implies the existence of mutual weak simulations between $K$ and $M^{\prime}$.

Example 10. Consider the work automaton $M^{\prime} \uparrow \sigma$ from Example 9, and let $\sim$ be the kernel that relates all states of $M^{\prime} \uparrow \sigma$. The contraction $\left(M^{\prime} \uparrow \sigma\right) / \sim$ results in $K$, as defined in Example 5 (modulo some irrelevant idling transitions). Define $\alpha\left(p,\left[\left(q_{1}, q_{2}\right)\right]\right)=\min H$, where $H=\left\{\left(q_{1}, q_{2}\right) \in\{0,1,2\}^{2} \mid p \models I_{\sigma}\left(q_{1}, q_{2}\right)\right\}$ is ordered by $\left(q_{1}, q_{2}\right) \leq\left(q_{1}^{\prime}, q_{2}^{\prime}\right)$ iff $q_{1} \leq q_{1}^{\prime}$ and $q_{2} \leq q_{2}^{\prime}$. By Theorem 2 , we have $M^{\prime} \preceq K$ and $M \preceq M^{\prime}$. By Example $7, M^{\prime}$ and $K$ are not weakly bisimilar.

The work automaton in Fig. 3 and the geometric representation of its infinite semantics in Fig. 4(a), only indirectly define a mutual exclusion protocol in $M^{\prime}$. By Example 10, we conclude that $M^{\prime}$ is weakly language equivalent to a much simpler work automaton $K$ that explicitly defines a mutual exclusion protocol by means of its state-invariant. Having such an explicit dependency visible in a state-invariant, reveals interesting behavioral properties of $M^{\prime}$, such as existence of non-blocking paths. These observations may be used to generate schedulers that force the execution to proceed along these non-blocking paths, which would enable a lock-free implementation and/or execution.

\section{Related Work}

Work automata without jobs correspond to port automata [12], which is a dataagnostic variant of constraint automata [3]. In a constraint automaton, each synchronization constraint $N \subseteq P$ is accompanied with a data constraint that interrelates the observed data $d_{a}$, at every port $a \in N$. Although it is straightforward to extend our work automata with data constraints, we refrain from doing 
so because our work focuses on synchronization rather then data-aware interaction. Hiding on constraint automata defined by Baier et al. in [3] essentially combines our hiding operator in Definition 9 with contraction from Theorem 2.

The syntax of work automata is similar to the syntax of timed automata [1]. Semantically, however, timed automata are different from work automata because jobs in a work automaton may progress independently (depending on whether or not they are scheduled to run on a processor), while clocks in a timed automaton progress at identical speeds. For the same reason, work automata differ semantically from timed constraint automata [2], which is introduced by Arbab et al. for the specification of time-dependent connectors.

This semantic difference suggests that we may specify a concurrent application as a hybrid automaton [11], which can be seen as a timed automaton wherein the speed of each clock, called a variable, is determined by a set of first order differential equations. Instead of fixing the speed of each process beforehand, via differential equations in hybrid automata, our scheduling approach aims to determine the speed of each process only after careful analysis of the application. Therefore, we do not use hybrid automata to specify a concurrent application.

Weighted automata [5] constitute another popular quantitative model for concurrent applications. Transitions in a weighted automaton are labeled by a weight from a given semiring. Although weights can define the workload of transitions, weighted automata do not show dependencies among different concurrent transitions, such as mutual exclusion [8]. As a consequence, weighted automata do not reveal dependencies induced by a protocol like work automata do.

A geometric perspective on concurrency has already been studied in the context of higher dimensional automata, introduced by Pratt [14] and Van Glabbeek [6]. This geometric perspective has been successfully applied in [8] to find and explain an essential counterexample in the study of semantic equivalences [7], which shows the importance of their, and indirectly our, geometric perspective. A higher dimensional automaton is a geometrical object that is constructed by gluing hypercubes. Each hypercube represents parallel execution of tasks associated with each dimension. This geometrical view on concurrency allows inheritance of standard mathematical techniques, such as homology and homotopy, which leads to new methods for studying concurrent applications $[9,10]$.

\section{Conclusion}

We extended work automata with state-invariants and resets and provided a formal semantics for these work automata. We defined weak simulation of work automata and presented translation and contraction operators that can simplify work automata while preserving their semantics up to weak simulation. Although translation is defined for any shift $(\theta, \rho)$, the conditions in Theorem 1 prove bisimulation only if $\theta$ has a unique solution. In the future, we want to investigate if this condition can be relaxed - and if so, at what cost - to enlarge the class of applications whose work automata can be simplified using our transformations. 
Our gluing procedure in Example 5 associates a work automaton with a geometrical object, and Example 6 shows that this geometric view reveals interesting behavioral properties of the application, such as mutual exclusion and existence of non-blocking execution paths. This observation suggests our results can lead to smart scheduling that yields lock-free implementation and/or executions.

State-invariants and guards in work automata model the exact amount of work that can be performed until a job blocks. In practice, however, these exact amounts of work are usually not known before-hand. This observation suggests that the 'crisp' subset of the multidimensional real vector space defined by the state-invariant may be replaced by a density function. We leave the formalization of such stochastic work automata as future work.

\section{References}

1. Alur, R., Dill, D.L.: A theory of timed automata. Theor. Comput. Sci. 126, 183235 (1994)

2. Arbab, F., Baier, C., de Boer, F.S., Rutten, J.: Models and temporal logics for timed component connectors. In: Proceedings of SEFM, pp. 198-207 (2004)

3. Baier, C., Sirjani, M., Arbab, F., Rutten, J.: Modeling component connectors in Reo by constraint automata. Sci. Comput. Program. 61(2), 75-113 (2006)

4. Dokter, K., Jongmans, S.-S., Arbab, F.: Scheduling games for concurrent systems. In: Lluch Lafuente, A., Proença, J. (eds.) COORDINATION 2016. LNCS, vol. 9686, pp. 84-100. Springer, Cham (2016). doi:10.1007/978-3-319-39519-7_6

5. Droste, M., Kuich, W., Vogler, H.: Handbook of Weighted Automata. Springer Science \& Business Media. Springer, Heidelberg (2009)

6. van Glabbeek, R.J.: Bisimulation semantics for higher dimensional automata. Email message, July 1991. http://theory.stanford.edu/ rvg/hda

7. van Glabbeek, R.J.: On the expressiveness of higher dimensional automata. Theor. Comput. Sci. 356(3), 265-290 (2006)

8. van Glabbeek, R.J., Vaandrager, F.: The difference between splitting in $n$ and $n+1$. Inform. Comput. 136(2), 109-142 (1997)

9. Goubault, E., Jensen, T.P.: Homology of higher dimensional automata. In: Cleaveland, W.R. (ed.) CONCUR 1992. LNCS, vol. 630, pp. 254-268. Springer, Heidelberg (1992). doi:10.1007/BFb0084796

10. Gunawardena, J.: Homotopy and concurrency. In: Păun, B., Rozenberg, G., Salomaa, A. (eds.) Current Trends in Theoretical Computer Science, pp. 447-459. World Scientific (2001)

11. Henzinger, T.A.: The theory of hybrid automata. In: Inan, M.K., Kurshan, R.P. (eds.) Verification of Digital and Hybrid Systems, pp. 265-292. Springer, Heidelberg (2000)

12. Koehler, C., Clarke, D.: Decomposing port automata. In: Proceedings of SAC, pp. 1369-1373. ACM (2009)

13. Milner, R.: Communication and Concurrency, vol. 84. Prentice Hall, New York (1989)

14. Pratt, V.: Modeling concurrency with geometry. In: Proceedings of POPL, pp. 311322. ACM (1991) 